\title{
N-PSO: endmember extraction using advance particle swarm optimization for NLMM
}

\author{
OMPRAKASH TEMBHURNE* and DEEPTI SHRIMANKAR \\ Department of Computer Science and Engineering, Visvesvaraya National Institute of Technology, \\ Nagpur 440010, India \\ e-mail: owtembhurne@gmail.com
}

MS received 21 July 2017; revised 26 August 2017; accepted 4 September 2017; published online 17 July 2018

\begin{abstract}
This paper presents a fully unsupervised endmember extraction technique for hyperspectral image unmixing using nonlinear mixing model. The underlying idea of the model is that the pixel reflectances are nonlinear functions of pure spectral components contaminated by an additive noise. These nonlinear functions are approximated using polynomial functions, leading to a polynomial post-nonlinear mixing model (PPNM). In an unknown environment, the evaluation of the parameters involved in PPNM model is a tedious task, which is categorized as an NP hard problem. A method based on the combination of swarm intelligence, least-square (LS) and sub-gradient-based optimization ( $\mathrm{SO}$ ) is proposed to estimate the parameters involved in the model. The particle swarm optimization (PSO) is used to search the optimal endmember combination in the feasible solution space. The nonlinearity and respective abundances are evaluated using the LS and SO method, respectively. The proposed method is equipped with an adaptive tuning parameter-free mechanism and modified updating strategy. This strategy not only improves the result in terms of overall accuracy but also maintains physical constraints on the value of the resultant endmember set. The proposed method has been evaluated using simulated and real hyperspectral scenes. The experimental results on the hyperspectral scenes show that the proposed method obtains a higher extraction precision than those of the existing endmember extraction algorithms. Statistical analysis on a real hyperspectral image shows that the results obtain using N-PSO are 20-40\% better than those from the existing approaches.
\end{abstract}

Keywords. Spectral unmixing; endmember extraction algorithms (EEA); particle swarm optimization (PSO); minimum volume simplex analysis; remote sensing; nonlinear mixing model (NLMM).

\section{Introduction}

Hyperspectral image [1] is a set of images concerned with capturing the reflectances across the electromagnetic spectrum. These bands typically span near-visible and infrared spectrum, although some sensors bands have shortwavelength infrared ranges, mid-wavelength infrared ranges and also thermal infrared ranges. The hyperspectral image capturing instruments such as the Airborne Visible Infra-Red Imaging Spectrometer (AVIRIS), and many more, can record the visible and near infra-red spectrum (from 0.4 to $2.5 \mu \mathrm{m}$ ). These images contain the information of an area that is $2-12 \mathrm{~km}$ wide and several kilometres long and also 224 bands of the continuous time interval [2]. Researchers use the hyperspectral scenes for identification of minerals, vegetation analysis, geographical survey, disaster management and identification of targets in the military applications. The analysis and interpretation of

*For correspondence hyperspectral scenes have become a viable area of research due to its real-time applications.

Satellite hyperspectral images contain data of large geographic areas. Due to the low spatial resolution of sensors and asymmetrical geometric conditions, pixels in images often contain a mixture of more than two endmembers. Multiple interactions among the scattering photons also add a mixing effect in the remotely sensed hyperspectral scenes. Radioactive transfer (RT) theory contributes in calculations of these mixing effects. However, a mathematical calculation of mixing effects using this theory is a tedious task for a real-time application [3].

Many researchers have made an attempt to solve the problem of mathematical tractability of RT theory by way of using the principle of approximate analysis. The approximate analysis is formulated in the form of various mixing models, which are explained in detail in [4]. The mixing effects (nonlinearity) due to various geometric conditions are explained by Dobigeon et al [5]. If features captured by a hyperspectral image are macroscopic and linearly separable, linear mixing model (LMM) 
demonstrates remarkable results. Similarly, when features are microscopic, multilayered, intimate and linearly nonseparable, the generalized bilinear model (GBM) and polynomial post-nonlinear mixing model (PPNM) yield remarkable results [5]. However, due to its versatility to the wide class of mixing models, PPNM model is used by most of the researchers for hyperspectral unmixing [5].

The spectral unmixing in NLMM involves the separation of the reflected spectrum into endmember spectra, their relative abundances and the nonlinearity parameter. The endmembers are considered to be pure pixels present in the scene, the abundances are their relative proportions and nonlinearity parameter is the unfortunate mixing effect. The endmember selection is a key step, which may cause an adverse effect on the qualitative performance. Hence the endmember extraction has a vital importance in the spectral unmixing.

Many endmember extraction algorithms (EEA), such as pixel purity index (PPI) [6], N-FINDR [7], vertex component analysis (VCA) [8], and the simplex growing algorithms such as MVSA [9], robust minimum enclosing simplex (RMVES) [10] and simplex identification via split augmented Lagrangian (SISAL) [11, 12], were proposed using LMM. To improve the precision of the endmember extraction, new evolutionary algorithms, such as ant colony optimization (ACO) [13], discrete particle swarm optimization (D-PSO) [14, 15] and adaptive differential evolution endmember extraction (ADEE) [16], were also proposed. However, this algorithm has several other drawbacks, i.e., the results of these algorithms depend on the proper setting of the adjustable parameters, the objective function designed by considering LMM only, unable to maintain the constraints on the values of endmember set. These algorithms will have to be run multiple times with different settings of the parameters to obtain their appropriate values.

The NLMM-based endmember extraction using distance geometry (DMaxD) is explained in [17]. While calculating the distance using PPNM model, the nonlinearity parameter is considered as a known parameter, which inclueds the DMaxD algorithm as a semi-supervised algorithm. A kernel-based NLMM and EEAs for hyperspectral unmixing is proposed in [18], where the nonlinear function belongs to a Hilbert space of vector-valued functions. Ammanouil et al [18] consider that the nonlinear contributions are dominant in some parts of the spectrum and are less pronounced in other parts. Therefore, instead of applying the same nonlinearity throughout the image, we modify nonlinearity parameter into a nonlinear diagonal matrix that contains the nonlinearity related to all the pixels from the image. The LMM-based robust linear mixing model (rLMM) and subsequent unmixing procedure using robust non-negative matrix factorization (rNMF) is introduced by Fevotte and Dobigeon [19]. However, this approach does not follow all the constraints on the values of the endmember set. Despite much advancement in the field of hyperspectral unmixing, the parameter-free, fully constrained, unsupervised, hyperspectral unmixing is always a challenging problem for NLMM.

Improvement in various meta-heuristic approaches has been achieved recently for various applications. In [20], a novel variant of Chemical Reaction Optimization (CRO) algorithm that combines the features of CRO and tabu search (TS) was proposed and named as CROTS algorithm. The particle swarm optimization-composite particle (PSOCP) and PSO are used to solve the problem of two-staged transportation [21]. Both the approaches were used to solve the problem of bulk wheat transportation and storage in India. Self-learning particle swarm optimization (SLPSO) and PSOCP are used to solve the problem of four-level food grain shipment in India [22]. To tackle the food grain supply chain problem of the Public Distribution System (PDS) in India, an effective meta-heuristic strategy called Improved Max-Min Ant System (IMMAS) was proposed in [23]. The effective meta-heuristic named PSOCP was proposed to solve the ship routing and scheduling problem, and maritime inventory routing problem [24-26]. Self-learning strategy, namely SLPSO, was used in [27] to solve the Vehicle Routing Problems (VRPs) in distribution centres with crossdocking operations. The applicability and innovation of the proposed N-PSO algorithm is inspired by the aforementioned meta-heuristic strategies.

In this paper, we have proposed a new approach of an unsupervised adaptive endmember extraction using N-PSO and updated PPNM model. It is fully constrained, independent of the manual selection of the tuning parameters. The tuning parameters are updated in each iteration based on the values of an objective function, which follow the feedback mechanism. The constraints are managed by clipping operations, which help the next generation to follow the search in an efficient manner. Instead of using a constant nonlinearity parameter throughout the image, the concept of a nonlinearity matrix that contains the nonlinearity parameter of every pixel has been used. Rather than random initialization, the MVSA algorithm or the addition or deletion of values of a specific range into the result of MVSA has been used to generate the initial population of endmembers. This helps the N-PSO to search in the proper direction and thereby improves the accuracy and the time complexity. Each endmember is an array of values; therefore the steps of particles velocity update have been modified accordingly.

Subsequently, section 2 describes the methods and materials used for the proposed approach. Section 3 describes the detailed methodology of proposed N-PSO. Section 4 contains the experimental results on simulated and real hyperspectral images, while section 5 provides the conclusion and prospective work. 


\section{Methods and materials}

\subsection{NLMM and endmember extraction problem}

The PPNM model is a representative model of NLMM. In modified PPNM model, the spectrum of mixed pixels in a hyperspectral scene is assumed to be a combination of deterministic endmembers $(\mathrm{E})$, their respective abundances $(\mathrm{s})$, and nonlinearity parameter $(\mathcal{E})$ in the form of a diagonal matrix which is related to all pixels from the image such that

$$
\mathrm{f}(\mathrm{E}, \mathrm{s}, \mathcal{E})=\mathrm{Es}+\mathcal{E}(\mathrm{Es}) \bullet(\mathrm{Es})+\mathrm{n}
$$

where (ANC) $s \geq 0,\left(\right.$ ASC) $\sum_{j=1}^{K} s_{j}=1, E \geq 0, E \leq 1$, the addition of Gaussian noise is described by the random vector (n), $K$ is the total number of endmembers and $(\bullet)$ is the Hadamard product.

The reconstruction error (RE) (in Eq. (2)) between the original image $(y)$ and the reconstructed image $(\tilde{y})$ is usually used to evaluate the correctness of the result, where $M$ is the total number of pixels available in the image, $\|\bullet\|$ is the $\ell_{2} \operatorname{norm},(\tilde{\mathrm{y}}=\tilde{\mathrm{E}} \times \tilde{\mathrm{s}}),(\tilde{\mathrm{E}})$ and $(\tilde{\mathrm{s}})$ are reconstructed endmembers and reconstructed abundances, respectively, and $L$ is the total number of available bands:

$$
\mathrm{RE}=\sqrt{\frac{1}{\mathrm{~L} \times \mathrm{M}}\left(\|\mathrm{y}-\tilde{\mathrm{y}}\|^{2}\right)} .
$$

For the simulated scenes, where the actual endmembers are known in advance, the spectral angle (SAM) (in Eq. (3)) between the original endmembers (E) and reconstructed endmembers $(\tilde{\mathrm{E}})$ is also used as an evaluation parameter, where $\left(\tilde{\mathrm{E}}^{\mathrm{T}}\right)$ is its transpose:

$$
\mathrm{SAM}=\cos ^{-1}\left(\frac{\tilde{\mathrm{E}}_{\mathrm{i}}^{\mathrm{T}} \mathrm{E}_{\mathrm{i}}}{\left\|\tilde{\mathrm{E}}_{\mathrm{i}}\right\|\left\|\mathrm{E}_{\mathrm{i}}\right\|}\right) .
$$

Assume that the value of the total number of endmembers $(K)$ is known in advance (i.e., the value extracted by HySime algorithm [28]). The endmembers extraction problem is described by the minimization problem with the objective function described in Eq. (4). The design variables are the values of endmembers. Their respective abundances and nonlinearity parameters are evaluated by the sub-gradient-based optimization (SO) and least-square (LS) algorithms presented in [29]. One of the sets of endmember among the population is initialized using MVSA algorithm for fast convergence.

$$
\operatorname{fun}(E)=\arg \min _{E}\left\{\frac{1}{2}\|y-\mathrm{f}(\mathrm{E}, \mathrm{s}, \mathcal{E})\|\right\}
$$

\subsection{PSO for estimating $E$}

PSO is a swarm intelligence approach inspired by the social foraging behaviour of a flock of birds [30]. The objective function of PSO is to improve the velocity and direction of a flock of birds. Thereby, they can find the optimal path towards the historically good areas of their environment in a multi-dimensional hyper-volume. It is a population-based optimization technique that considers the random population of the solutions to proceed for a globally acceptable solution. The population is the total number of random solutions generated in the solution space. In PSO, the improvement in the position of the particle is influenced by its own velocity, the best known global position and velocity and the local best position and velocity. The improvement in knowledge of the particle is a continuous process. The endmembers (E) are the considered particles. The feedback mechanism takes care of the assurance of improvement in the knowledge update. This process continues until the group of solutions does not reach the global optimum solution.

\subsection{LS method for estimating $E$}

The LS method related to Eq. (1) consists in minimizing the following:

$$
\mathrm{J}(s, \mathcal{E})=\|\mathrm{y}-\mathrm{f}(\mathrm{E}, \mathrm{s}, \mathcal{E})\|^{2}=\|y-\mathrm{Es}+\mathcal{E}(\mathrm{Es}) \bullet(\mathrm{Es})+\mathrm{n}\|^{2}
$$

In Eq. (5) the known values $(E, n)$ are considered as constants; therefore, $J(s, E)$ is quadratic with respect to $E$. As a consequence, by differentiating Eq. (5) with respect to $\mathcal{E}$. the value of $\mathcal{E}$ can be obtained in closed form by Eq. (6):

$$
\mathcal{E}=\frac{(\mathrm{y}-\mathrm{Es})^{\mathrm{T}}(\mathrm{Es} \bullet \mathrm{Es})}{(\mathrm{Es} \bullet \mathrm{Es})^{\mathrm{T}}(\mathrm{Es} \bullet \mathrm{Es})}
$$

Substituting Eq. (6) in Eq. (5), the following equation (7) can be obtained where the only unknown parameter is $s$ :

$$
\mathrm{J}(s)=\left\|\mathrm{y}-\mathrm{Es}-\left(\frac{(\mathrm{y}-\mathrm{Es})^{\mathrm{T}}(\mathrm{Es} \bullet \mathrm{Es})}{(\mathrm{Es} \bullet \mathrm{Es})^{\mathrm{T}}(\mathrm{Es} \bullet \mathrm{Es})}\right)(\mathrm{Es}) \bullet(\mathrm{Es})-n\right\|^{2} .
$$

The unknown parameter $(s)$ is then computed by the SO method with a constrained objective function defined in Eq. (8):

$$
s=\arg \min _{s}\{J(s)\}
$$

Once $s$ (with ANC and ASC) has been computed, the value of $\mathcal{E}$ can be computed using Eq. (6). 


\subsection{SO for estimating $s$}

Equation (8) is solved by SO that is designed for the constrained problems. The ASC of the abundance $(s)$ is managed by expressing Eq. (8) as a function of $(s, K)$, where $\left(\mathrm{s}_{\mathrm{K}}=1-\sum_{\mathrm{r}=1}^{\mathrm{K}-1} \mathrm{~s}_{\mathrm{r}}\right)$. The SO algorithm performs a sequential line search operation along the direction $\left(d_{r}\right)$, which is calculated by taking the partial derivatives of Eq. (7) with respect to $s_{r}$ (where $r=1, \ldots, K-1$ ), i.e.

$$
\mathrm{d}_{\mathrm{r}}=-\frac{\partial}{\partial \mathrm{s}_{\mathrm{r}}}(J(s)) .
$$

Finally, Eq. (8) is solved by the line search procedure, whose upper bounds are defined by the rule given in Eq. (10), which ensures ASC and ANC (here $i=1, \ldots, M$ ):

$$
\mathrm{s}_{\mathrm{r}, \mathrm{i}}= \begin{cases}0 & \text { if } \mathrm{d}_{r}=0 \\ \mathrm{~s}_{\mathrm{r}} & \text { if } \mathrm{d}_{r}>0 \\ \mathrm{~s}_{\mathrm{r}}-\sum_{\mathrm{i}=1, \mathrm{i} \neq \mathrm{r}}^{\mathrm{K}-1} \mathrm{a}_{\mathrm{i}} & \text { if } \mathrm{d}_{r}<0\end{cases}
$$

The abundances are then updated iteratively until convergence.

\section{Proposed algorithm}

In N-PSO, the endmember extraction is a problem of combinatorial optimization. The original PSO algorithm is highly dependent on the selection of the proper value of the acceleration constants and the inertia weights. The values of these control parameters are problem dependent. It requires a lot of time to set the proper values of these parameters. This problem is avoided in the N-PSO, by adaptively setting the values of these parameters during the stages of the evolution.

The design parameters of the proposed optimization problem are a set of the $K$ endmembers, where each endmember is an array of size $L$. In addition, to decrease the search space and improve the computational efficiency, the MVSA algorithm is used to obtain endmembers in one of the initial population. To optimize the direction of search, the endmembers generated by MVSA algorithm are used to generate rest of the population by scaling the values of endmembers in positive or negative direction using Eq. (11):

$$
\mathrm{E}_{\{\mathrm{i}\}} \leftarrow \mathrm{E}_{\{\mathrm{MVSA}\}}+(\mathrm{a}+(\mathrm{b}-\mathrm{a})) \operatorname{rand}([\mathrm{L}, \mathrm{K}])
$$

where $(a+(b-a) \operatorname{rand}([L, K]))$ is used to scale the value of rest of the population by a magnitude between $a$ and $b$.

The N-PSO algorithm consists of three phases, namely, 'initialization phase', 'update phase' and 'termination phase'.

\subsection{Initialization phase}

- Initialize a population of endmembers $(E)$ of size $(N, K)$ using the MVSA and random approach using Eq. (11).

- Clip the initial population of endmember sets within the range of the physical constraints using Eq. (12):

$$
\mathrm{E}_{\mathrm{i}}=\max \left(E_{\mathrm{i}}, 0\right), \mathrm{E}_{\mathrm{i}}=\min \left(E_{\mathrm{i}}, 1\right) .
$$

- Initialize the inertia weight $\left(\omega_{i}\right)$, the inertia weight damping ratio $\left(\omega_{\mathrm{i}}^{\mathrm{d}}\right)$ and the acceleration constants $\left(c_{i}^{1} \& c_{i}^{2}\right)$ using any random variable between 0 and 1 .

- Calculate the values of the objective function of the entire population using the initial endmembers $(E)$, their abundances and nonlinearity parameter using SO and LS algorithms presented in [29].

\subsection{Adaptive updation using adaptive control parameters}

During this phase, the particles try to improve themselves and others to have the best value of the objective function. The endmembers are the actual parameters involved in the objective function of a given optimization problem. The best value of the objective function is the best solution. The parameter that generates the best solution in the entire population is considered as a global solution $\left(E_{\mathrm{g}}^{\text {best }}\right)$. The position and velocity of the remaining particles are updated using the local best $\left(E_{i}^{\text {best }}\right)$ particles. 


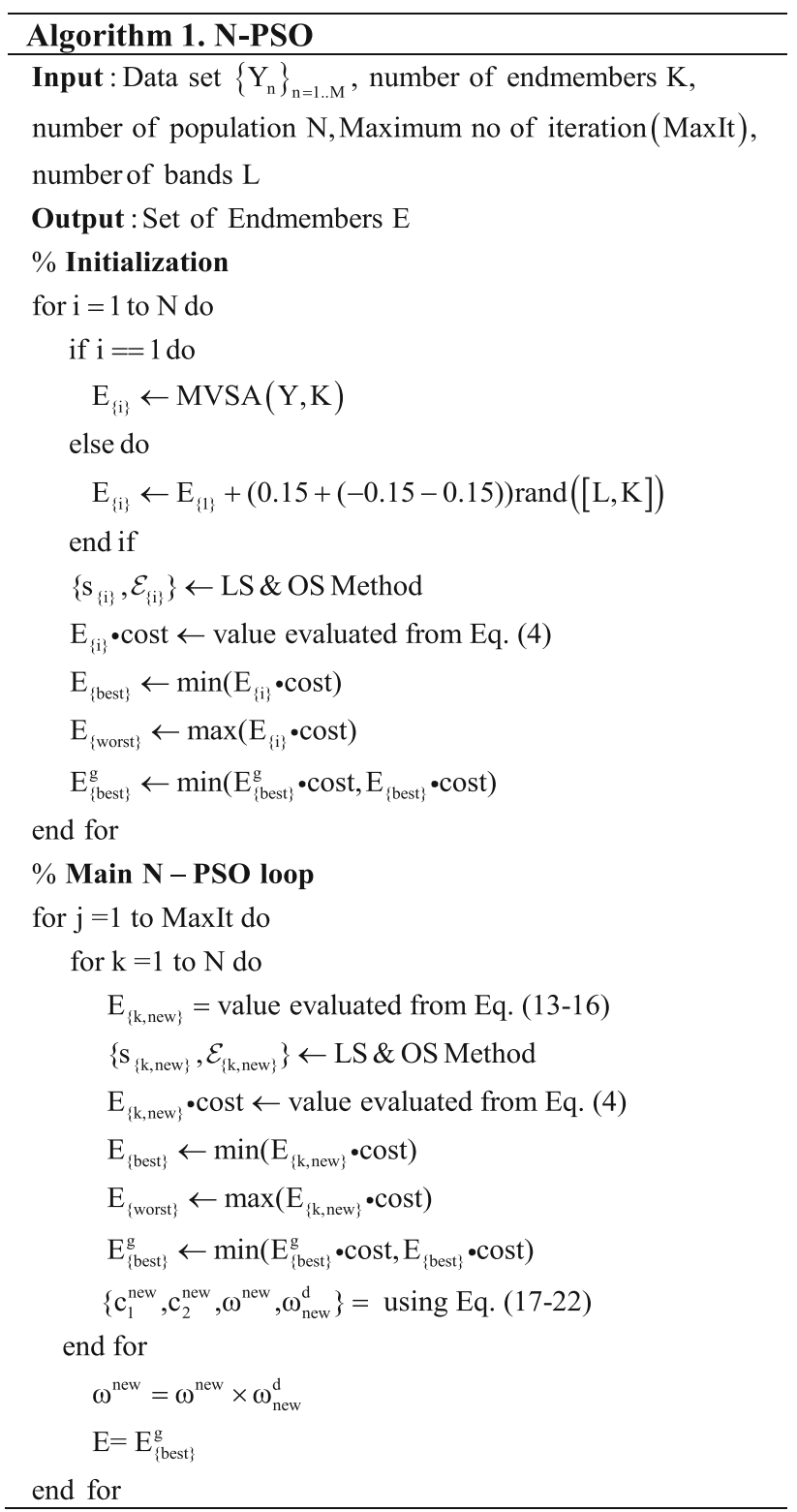

The following steps govern the updated rules with the adaptive control parameters.

Step 1. Arrange the endmember sets of the entire population $(N)$ in ascending order as per the objective function value. It is useful to select the neighbourhood particles from the population.

Step 2. The adaptive update and constraints management of the $i^{\text {th }}$ particle are described in Eqs. (13)-(16):

$$
\begin{gathered}
\left.\mathrm{v}_{\text {new }}=\omega_{\mathrm{i}} \mathrm{v}_{\mathrm{i}}+\mathrm{c}_{\mathrm{i}}^{1} \times \operatorname{rand}([L \times K])\right) \times\left(E_{\mathrm{i}}^{\text {best }}-\mathrm{E}_{\mathrm{i}}\right)+\cdots \mathrm{c}_{\mathrm{i}}^{2} \\
\times \operatorname{rand}([L \times K])) \times\left(E_{\mathrm{g}}^{\text {best }}-E_{\mathrm{i}}\right) \\
\mathrm{v}_{\text {new }}=\min \left(v_{\text {new }}, 0\right), \mathrm{v}_{\text {new }}=\max \left(\mathrm{v}_{\text {new }}, 1\right) \\
\mathrm{E}_{\text {new }}=\mathrm{E}_{\mathrm{i}}+\mathrm{v}_{\mathrm{i}}
\end{gathered}
$$

$$
\mathrm{E}_{\text {new }}=\min \left(E_{\mathrm{i}+1}, 0\right), \mathrm{E}_{\text {new }}=\max \left(E_{\mathrm{i}+1}, 1\right)
$$

where $v_{i}(i=1, \ldots, N)$ denotes the velocity of the $i^{\text {th }}$ particle; $\operatorname{rand}([L \times K])$ creates a random matrix of size $L K$ having values between 0 and $1 . E_{\text {new }}$ and $v_{\text {new }}$ are the updated values of endmember (i.e., particle) and particle velocity, respectively.

Step 3: Automatically update the values of the control parameters of the $i^{\text {th }}$ particle in $t^{\text {th }}$ generation (using Eqs. (17)-(22)):

$$
\begin{aligned}
& \mathrm{p}_{\mathrm{i}}=\frac{\operatorname{fun}\left(E_{\mathrm{i}}\right)-f u n\left(E_{\mathrm{g}}^{\text {best }}\right)}{f u n\left(E_{\mathrm{g}}^{\text {best }}\right)-f u n\left(E_{\mathrm{g}}^{\text {worst }}\right)} \\
& c_{\text {new }}^{1}= \begin{cases}\operatorname{rand}_{2} & \text { if } \text { rand }_{1}<p_{i} \\
c_{i}^{1} & \text { otherwise }\end{cases} \\
& c_{\text {new }}^{2}= \begin{cases}\operatorname{rand}_{4} & \text { if } \operatorname{rand}_{3}<p_{i} \\
c_{i}^{2} & \text { otherwise }\end{cases} \\
& \omega_{\text {new }}= \begin{cases}\operatorname{rand}_{6} & \text { if } \operatorname{rand}_{5}<p_{i} \\
\omega_{i} & \text { otherwise }\end{cases} \\
& \omega_{\text {new }}^{\mathrm{d}}= \begin{cases}\operatorname{rand}_{8} & \text { if } \operatorname{rand}_{7}<\mathrm{p}_{\mathrm{i}} \\
\omega_{\mathrm{i}}^{\mathrm{d}} & \text { otherwise }\end{cases} \\
& \omega_{\text {new }}=\omega_{\text {new }} \times \omega_{\text {new }}^{\mathrm{d}}
\end{aligned}
$$

where $\operatorname{rand}_{1}, \operatorname{rand}_{3}$ and $\operatorname{rand}_{6-8}$ are uniform random variables having values in the interval $(0,1)$, andrand ${ }_{2}, \operatorname{rand}_{4}$ are uniform random variables having values in the intervals $(0,1),(1,2)$, respectively. $\left(\mathrm{E}_{\mathrm{g}}^{\text {best }}, \mathrm{E}_{\mathrm{g}}^{\text {worst }}\right)$ is the global best and worst endmember set amongst the updated population.

Step 4: Update the values of the objective function of the entire population using the new endmembers, their respective new abundances and new nonlinearity factor using SO and LS method (as per Eq. (23)):

$$
\operatorname{fun}\left(\mathrm{E}_{\text {new }}\right)=\arg \min _{\mathrm{E}_{\text {new }}}\left\{\frac{1}{2}\left\|\mathrm{y}-\mathrm{f}\left(\mathrm{E}_{\text {new }}, \mathrm{s}_{\text {new }}, \varepsilon_{\text {new }}\right)\right\|\right\} .
$$

These steps use the feedback mechanism while updating the values of the endmembers by the new values using Eq. (23):

$$
E_{\text {new }}= \begin{cases}E_{\text {new }} & \text { if }\left(\text { fun }\left(E_{\text {new }}\right)<\text { fun }\left(E_{i}\right)\right) \\ E_{i} & \text { otherwise }\end{cases}
$$

\subsection{Termination phase}

Being an iterative process, the convergence of N-PSO is also a matter of concern. As the number of iterations $(t)$ increases, the reconstruction error [31] RE gradually reduces until it reaches the stable state. The maximum number of iteration is set to 100 . If the difference of the log-likelihood values of the objective function between two successive iterations is equal to zero after 10 attempts, we consider it as 


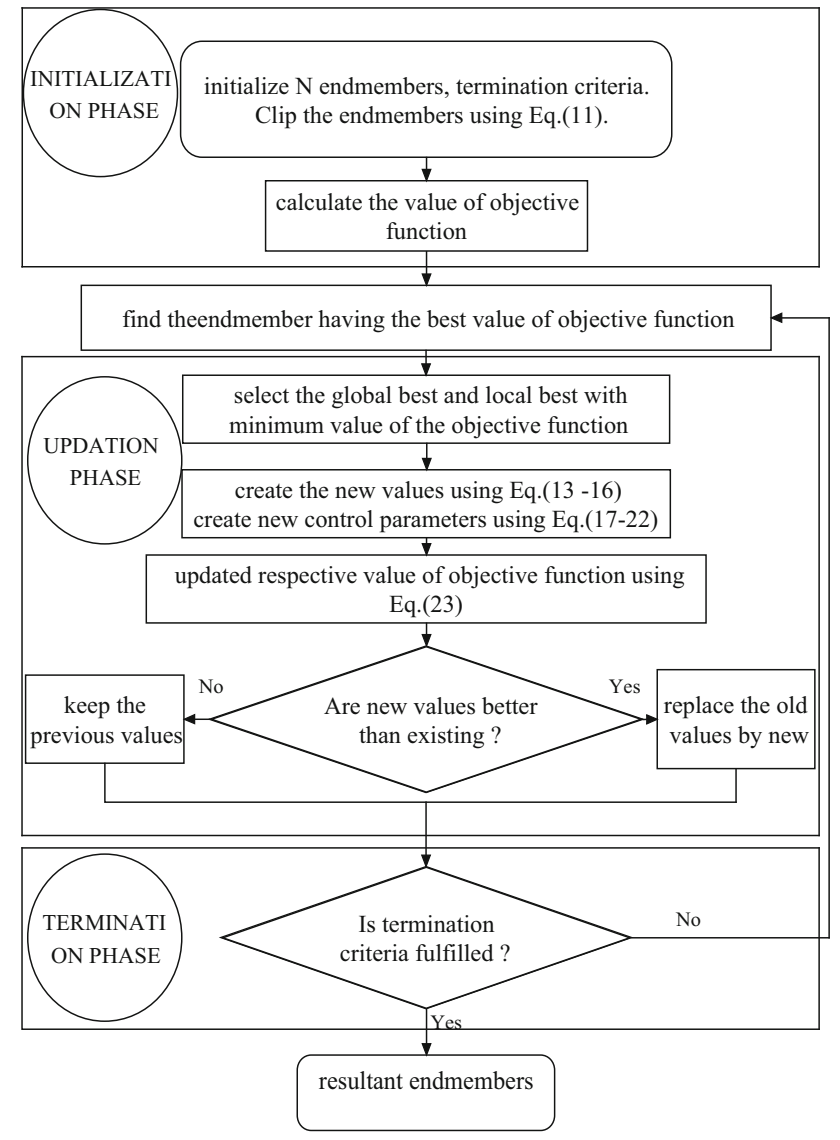

Figure 1. Flowchart of N-PSO algorithm.

a convergence situation. A flowchart showing the complete methodology of an N-PSO is illustrated in figure 1 .

\section{Experiments and analyses}

The experiments are conducted on two different types of hyperspectral images, i.e., simulated scenes and widely used real hyperspectral scenes such as Cuprite data set and Indian Pines data set.

Zero-mean white Gaussian noise $(e)$ is added to the synthetic pixel $(y)$ to evaluate the robustness to the variation in Signal-To-Noise ratio (SNR). The value of SNR is evaluated using Eq. (25):

$$
\mathrm{SNR}=10 \log _{10} \frac{E\left[y^{\mathrm{T}} y\right]}{E\left[e^{\mathrm{T}} e\right]}
$$

where $E[$.$] is the expectation maximization, y^{\mathrm{T}}$ and $e^{\mathrm{T}}$ are the transpose of the respective vectors.

As stated earlier, the proposed algorithm and other two state-of-art algorithms were implemented using Matlab R2013a on a 64-bit Intel Core i5 processor with Windows 7 operating system.

\subsection{Data set description}

Simulated scene: The simulated scene of size $60 \times 60 \times 224$ was synthesized using abundances created by Dirichlet_rnd function [32]; the endmembers were collected from USGS digital spectral library [33] and the nonlinearity parameter was random.

Cuprite data set: The data are captured by NASA's AVIRIS sensor over the Cuprite mining district in Nevada [34].

Indian Pines data set: Data set of size $145 \times 145 \times 200$ and its ground truth are captured over the Indian Pines test site in North-western Indiana [35].

\subsection{State-of-art algorithms}

VCA: The LMM-based EEA and its source code were collected from [36]. The synthetic image was created using LMM. The RE was calculated accordingly.

DMaxD: The NLMM-based EEA and its source code were collected from [37]. The synthetic image was created using LMM. The RE was calculated accordingly.

$r N M F$ : The rLMM-based EEA and its source code were collected from [38]. The synthetic image was created using rLMM. The RE was calculated accordingly.

\subsection{Adjustable parameter analysis}

The total number of population and the total number of iterations required for the convergence are adjustable parameters, which may have a distinct effect on the performance of the algorithm. Table 1 illustrates the effect of different population sizes on unmixing error and computing

Table 1. The RE and computing time by N-PSO and PSO algorithms when $N=3,8,14,30,40$ and 50.

\begin{tabular}{llccccc}
\hline & $N=3$ & $N=8$ & $N=14$ & $N=30$ & $N=40$ & $N=50$ \\
\hline RE (PSO) & 0.065 & 0.059 & 0.060 & 0.057 & 0.057 \\
Time (PSO) & 7.88 & 20.76 & 33.13 & 71.46 & 100.1 & 126.83 \\
RE (N-PSO) & 0.065 & 0.056 & 0.053 & $\mathbf{0 . 0 4 7}$ & 0.046 \\
Time (N- & 6.57 & 16.2 & 25.24 & $\mathbf{4 7 . 8 3}$ & 68.44 \\
$\quad$ PSO) & & & & 84.22
\end{tabular}

Best solutions are in bold. 
Table 2. The performance of N-PSO with other state-of-art algorithms when $K=3,7,9,12$ and 15 .

\begin{tabular}{|c|c|c|c|c|c|c|c|c|c|c|}
\hline \multirow[b]{2}{*}{$\mathrm{SNR}=50 \mathrm{~dB}$} & \multicolumn{2}{|c|}{$K=3$} & \multicolumn{2}{|c|}{$K=7$} & \multicolumn{2}{|c|}{$K=9$} & \multicolumn{2}{|c|}{$K=12$} & \multicolumn{2}{|c|}{$K=15$} \\
\hline & $\mathrm{RE}$ & SAM & $\mathrm{RE}$ & SAM & $\mathrm{RE}$ & SAM & $\mathrm{RE}$ & SAM & $\mathrm{RE}$ & SAM \\
\hline VCA [8] & 0.1885 & 0.1484 & 0.1049 & 0.1946 & 0.2834 & 0.3562 & 0.1475 & 0.4574 & 0.0914 & 0.4864 \\
\hline DMaxD [17] & 0.1517 & 0.1206 & 0.1975 & 0.2528 & 0.2036 & 0.2869 & 0.1241 & 0.4255 & 0.1071 & 0.5550 \\
\hline rNMF [19] & 0.1091 & 0.2188 & 0.0735 & 0.4321 & 0.1412 & 0.4356 & 0.0536 & 0.4886 & 0.1245 & 0.5373 \\
\hline N-PSO & 0.1970 & 0.1288 & 0.0946 & 0.1450 & 0.1760 & 0.1458 & 0.0945 & 0.4039 & 0.1021 & 0.3201 \\
\hline
\end{tabular}

Best solutions are in bold.

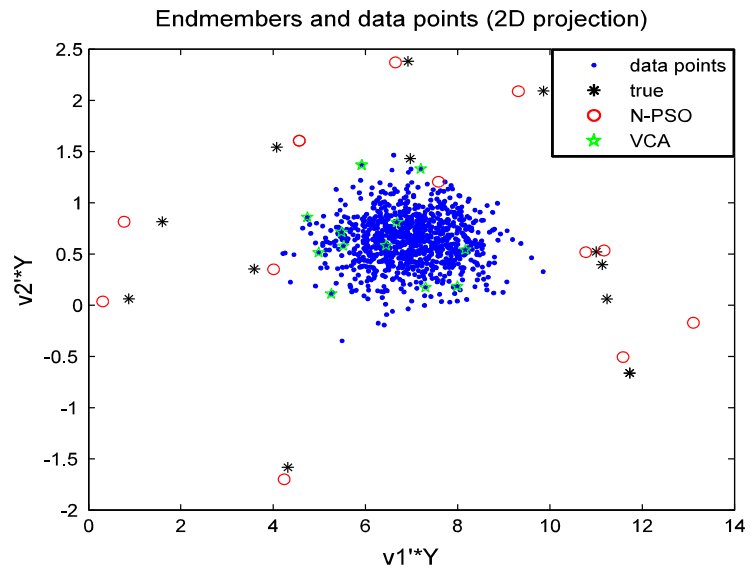

Figure 2. The data set and endmembers (2D) projection when $K=12$ and $\mathrm{SNR}=50 \mathrm{~dB}$.

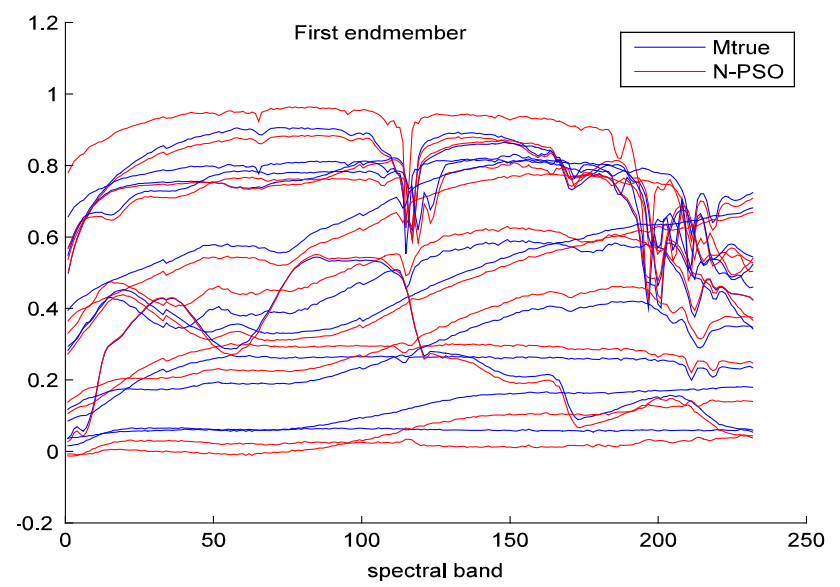

Figure 3. The true endmembers and endmembers extracted by $\mathrm{N}$-PSO when $K=12$ and $\mathrm{SNR}=50 \mathrm{~dB}$. time (note: the analysis was performed for a single pixel). When the population size is more than 14, the unmixing errors have small variations in case of N-PSO. However, the unmixing error does not change a lot in case of traditional PSO. The time required to execute traditional PSO is always greater than that of the N-PSO. The increase in the population also leads to more computing time. In this context, it is worthwhile to say that the population size of 30 is quite abreast of the unmixing errors like that of larger population size. In the experiment, we set the maximum iterations required for convergence to 100 .

\subsection{Experiment 1-simulated scene}

Three different types of the experiments have been performed on the synthetic images to evaluate the performance of the proposed method, which are the following:

1. with the increase in spectral variability,

2. under the influence of noise and

3 . in known and unknown environments.

The total number of the endmembers used is directly related to the spectral variability. Therefore, the performance of proposed algorithm was evaluated using the simulated scenes, which were synthesized by a different combination of endmembers (from $K=3$ to 15 and SNR = $50 \mathrm{~dB}$ ). The performance of three different EEAs associated to the simulated scenes is illustrated in table 2. N-PSO algorithm has better RE and SAM compared with the other state-of-art algorithms except for rNMF when $K=3,7,9$ and 12. However, the observed difference in RE of N-PSO and $\mathrm{rNMF}$ is very small and the SAM of rNMF is at its worst value. The $2 \mathrm{D}$ scatter plot of the endmembers and data points when $K=12$ is shown in figure 2. A graph

Table 3. The performance of N-PSO with other state-of- art algorithms when SNR =15, 25, 35, 60 and $\infty \mathrm{dB}$.

\begin{tabular}{|c|c|c|c|c|c|c|c|c|c|c|}
\hline \multirow[b]{2}{*}{$K=3$} & \multicolumn{2}{|c|}{$\mathrm{SNR}=15 \mathrm{~dB}$} & \multicolumn{2}{|c|}{$\mathrm{SNR}=25 \mathrm{~dB}$} & \multicolumn{2}{|c|}{$\mathrm{SNR}=35 \mathrm{~dB}$} & \multicolumn{2}{|c|}{$\mathrm{SNR}=60 \mathrm{~dB}$} & \multicolumn{2}{|c|}{$\mathrm{SNR}=\infty \mathrm{dB}$} \\
\hline & $\mathrm{RE}$ & SAM & $\mathrm{RE}$ & SAM & $\mathrm{RE}$ & SAM & $\mathrm{RE}$ & SAM & $\mathrm{RE}$ & SAM \\
\hline VCA & 0.2902 & 0.1263 & 0.1458 & 0.1810 & 0.0561 & 0.0456 & 0.1598 & 0.1656 & 0.1605 & 0.1167 \\
\hline DMaxD & 0.1153 & 0.1053 & 0.1195 & 0.1556 & 0.0764 & 0.0573 & 0.0336 & 0.1156 & 0.0776 & 0.0854 \\
\hline $\mathrm{rNMF}$ & 0.1001 & 0.0634 & 0.1340 & 0.6157 & 0.0690 & 0.0794 & 0.0573 & 0.2418 & 0.0343 & 0.2161 \\
\hline N-PSO & 0.1029 & $\mathbf{0 . 0 3 0 7}$ & 0.1230 & 0.1698 & 0.0412 & 0.1110 & 0.0124 & 0.0792 & 0.0076 & 0.0543 \\
\hline
\end{tabular}

Best solutions are in bold. 


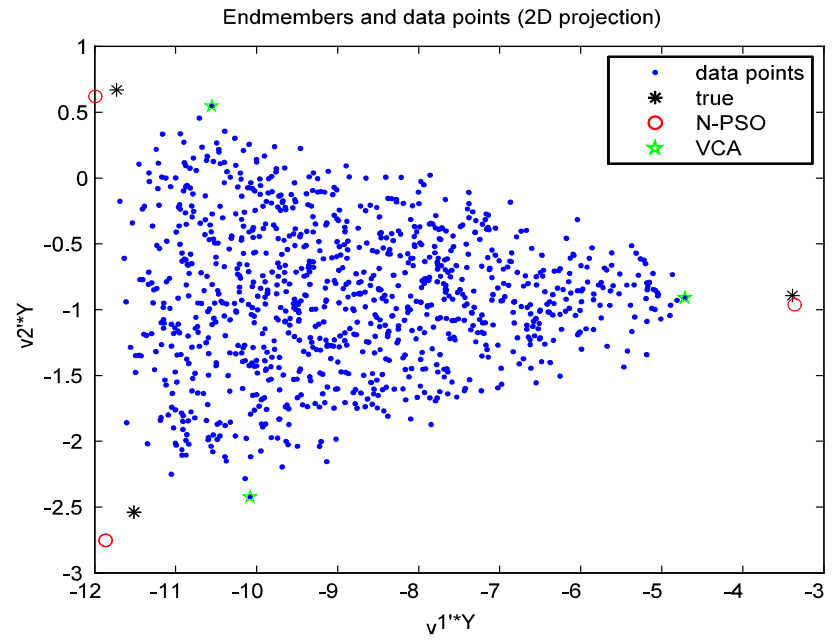

Figure 4. The data set and endmembers (2D) projection when $K=3$ and $\mathrm{SNR}=15 \mathrm{~dB}$.

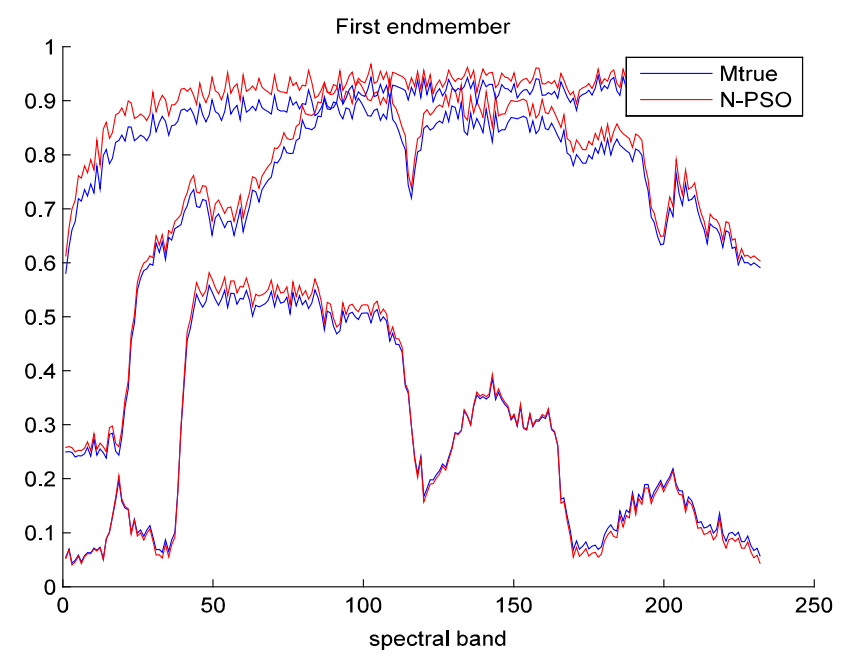

Figure 5. The true endmembers and endmembers extracted by $\mathrm{N}-\mathrm{PSO}$ when $K=3$ and $\mathrm{SNR}=15 \mathrm{~dB}$.

showing the similarity between the true endmembers and the endmembers extracted by N-PSO is illustrated in figure 3. The endmembers evaluated by the N-PSO are far near to the true endmembers. These experiments show the superiority of the N-PSO algorithm over other state-of-art algorithms.
Another experiment was performed to evaluate the performance of the proposed method under the influence of noise. The simulated scene of three endmembers is used for the evaluation. A zero-mean white Gaussian noise (from SNR 15 to $\infty \mathrm{dB}$ ) is added to the simulated scene. The performance of three different EEAs for the different values of SNR is illustrated in table 3. N-PSO algorithm has better RE and SAM compared with the other state of art algorithms except for $\mathrm{rNMF}$ and DMaxD when SNR $=15$ and $25 \mathrm{~dB}$, respectively. However, it is a notable thing that the observed difference is very small. The $2 \mathrm{D}$ scatter plot of the endmembers and data points when $K=3$ and SNR $=15 \mathrm{~dB}$ are shown in figure 4. A graph showing the similarity between the true endmembers and the endmembers extracted by N-PSO is illustrated in figure 5 . The results show the robustness of N-PSO with SNR varying from 15 to $\infty \mathrm{dB}$.

To evaluate the performance of the proposed algorithm in the known and unknown environments, a synthetic image with 14 endmembers is used. The unmixing results of the N-PSO and other three algorithms are given in table 3. In particular, when an exact number (real) of the endmembers are known, all the algorithms show good results. The RE of rNMF is better than those of other approaches when $K=7$ and 9. Although the rNMF algorithm satisfies the nonnegative constraint, the maximum value of the endmember in most of the cases is greater than one, which creates errors in construction of the synthetic image. The results from table 4 show that the N-PSO achieves better accuracy irrespective of the knowledge of the exact numbers of endmembers contributed in the spectral unmixing. Besides, the values of the endmember extracted by N-PSO are within the limit of the physical constraints.

To evaluate the performance of N-PSO, comparison of $\mathrm{N}$-PSO to the existing heuristic approaches is an important issue. Since N-PSO is the first heuristic approach designed for NLMM, the performance of N-PSO is compared only to that of the traditional PSO. The results of comparisons of the different values of population are enlisted in table 1 . The RE of N-PSO algorithm is better than that of traditional PSO. The results from the experiments conducted with a different population size show the superiority of N-PSO algorithm over traditional PSO.

Table 4. The performance of N-PSO with other state-of-art algorithms in known and unknown environments when image was created using $K($ real $)=15$.

\begin{tabular}{|c|c|c|c|c|c|c|c|c|c|c|}
\hline \multirow[b]{2}{*}{$\mathrm{SNR}=50 \mathrm{~dB}$} & \multicolumn{2}{|c|}{$K=3$ (unknown) } & \multicolumn{2}{|c|}{$K=7$ (unknown) } & \multicolumn{2}{|c|}{$K=9$ (unknown) } & \multicolumn{2}{|c|}{$K=12($ unknown $)$} & \multicolumn{2}{|c|}{$K=14$ (real) } \\
\hline & $\mathrm{RE}$ & $(\min , \max )$ & $\mathrm{RE}$ & $(\min , \max )$ & $\mathrm{RE}$ & $(\min , \max )$ & $\mathrm{RE}$ & $(\min , \max )$ & $\mathrm{RE}$ & $(\min , \max )$ \\
\hline VCA & 1.296 & $(0.2200,0.7014)$ & 0.122 & $(0.2134,0.7324)$ & 0.229 & $(0.2202,0.7277)$ & 0.091 & $(0.21$ & 0.071 & $(0.1817,0.6922)$ \\
\hline DMaxD & 0.291 & $(0.1684,0.7954)$ & 0.122 & $(0.2533,0.8184)$ & 0.071 & $(0.1747,0.7275)$ & 0.105 & $(0.2211,0.7286)$ & 0.104 & $(0.1817,0.7229)$ \\
\hline rNMF & 0.126 & $(0.1508,0.8932)$ & 0.099 & $(0.1288,1.1204)$ & 0.070 & $(0.0717,1.2891)$ & 0.067 & $(0.0826, \mathbf{1 . 2 0 9 7})$ & 0.062 & $(0.0548, \mathbf{1 . 1 1 5})$ \\
\hline N-PSO & 0.112 & $(0,1)$ & 0.101 & $(0,1)$ & 0.109 & $(0,1)$ & $\mathbf{0 . 0 3 0}$ & $(0.0152,0.9643)$ & 0.044 & $(0.0401,0.9131)$ \\
\hline
\end{tabular}

Best solutions are in bold. 


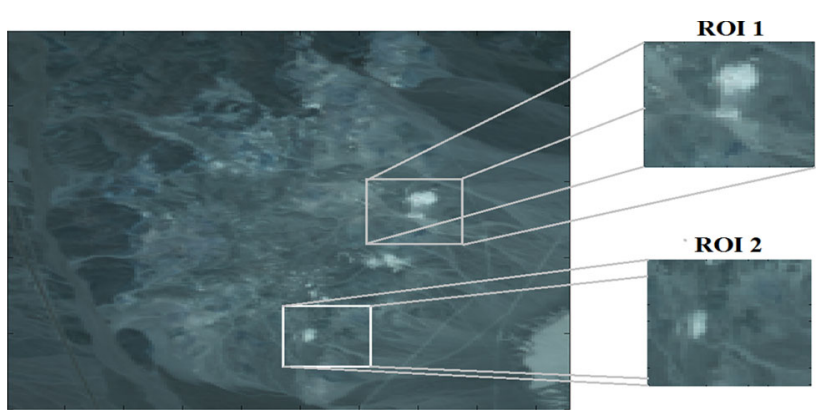

Figure 6. False colour composition of Cuprite data set with bands [13 54 97] (left) and region of interest (right).

Table 5. Performance of N-PSO with other EEAs using the Cuprite data set.

\begin{tabular}{lcccc}
\hline$K=14$ & RE (ROI & RE (ROI & Time (ROI & Time (ROI \\
& $1)$ & $2)$ & $1)$ & $2)$ \\
\hline VCA & 0.1107 & 0.1581 & 0.51 & 0.49 \\
DMaxD & 0.1568 & 0.4937 & 8.75 & 8.12 \\
rNMF & 0.1038 & 0.5995 & 37.67 & 37.75 \\
N-PSO & $\mathbf{0 . 0 4 5 1}$ & $\mathbf{0 . 0 3 1 2}$ & $\mathbf{5 6 0}$ & $\mathbf{5 4 7}$ \\
\hline
\end{tabular}

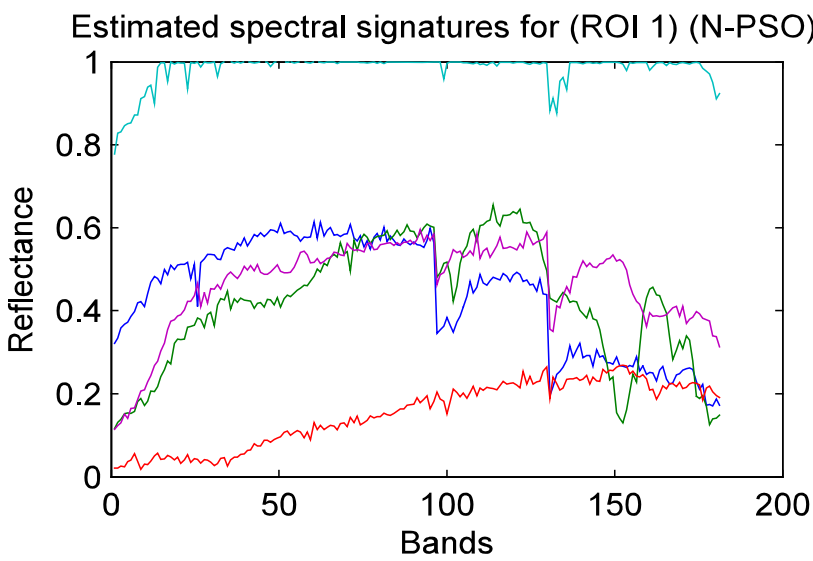

Figure 7. Estimated spectral signature for ROI 1 using N-PSO.

The time required to execute the N-PSO and traditional PSO for different combinations of endmembers is also shown in table 1 . As the number of population increases, the time required to execute for both algorithms increases. Instead of the addition of extra steps, the time required to execute N-PSO is less than that of traditional PSO because of the faster convergence.

\subsection{Experiment 2-real hyperspectral scene}

The second experiment is accomplished using Cuprite data set as shown in figure 6. Two regions of interest (ROI) are

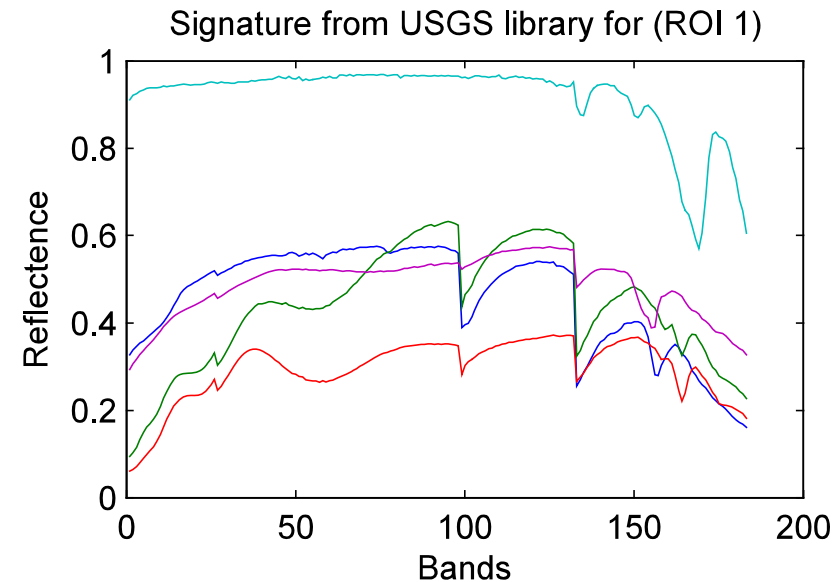

Figure 8. Matched signatures from USGS spectral library for ROI 1.

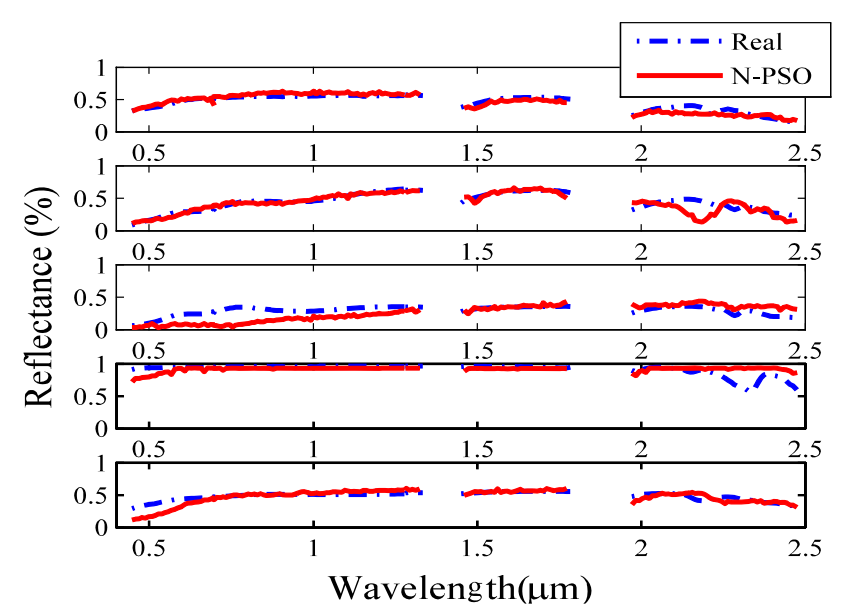

Figure 9. Graph showing similarity between the endmember signatures of real (extracted from USGS) and that extracted by N-PSO for ROI 1.

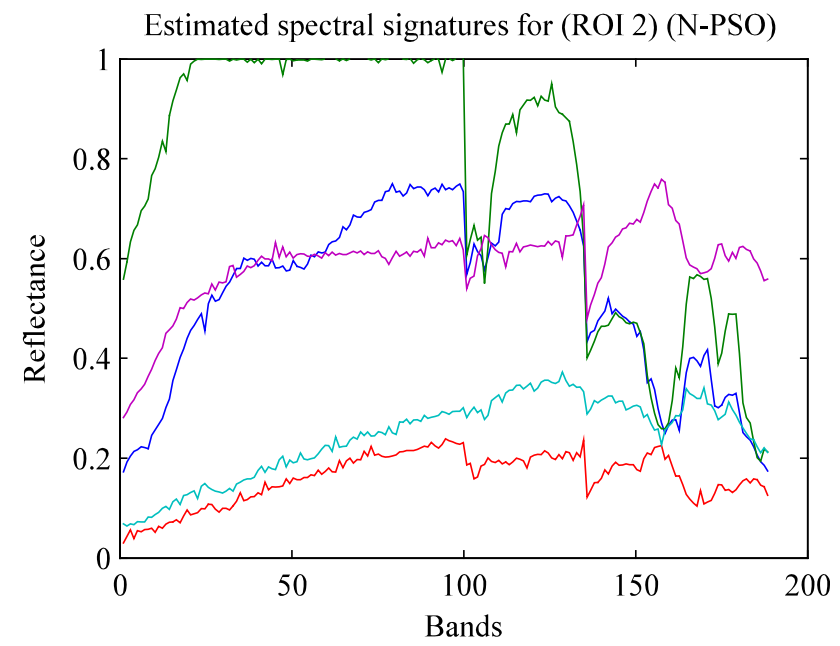

Figure 10. Estimated spectral signature for ROI 2 using N-PSO. 


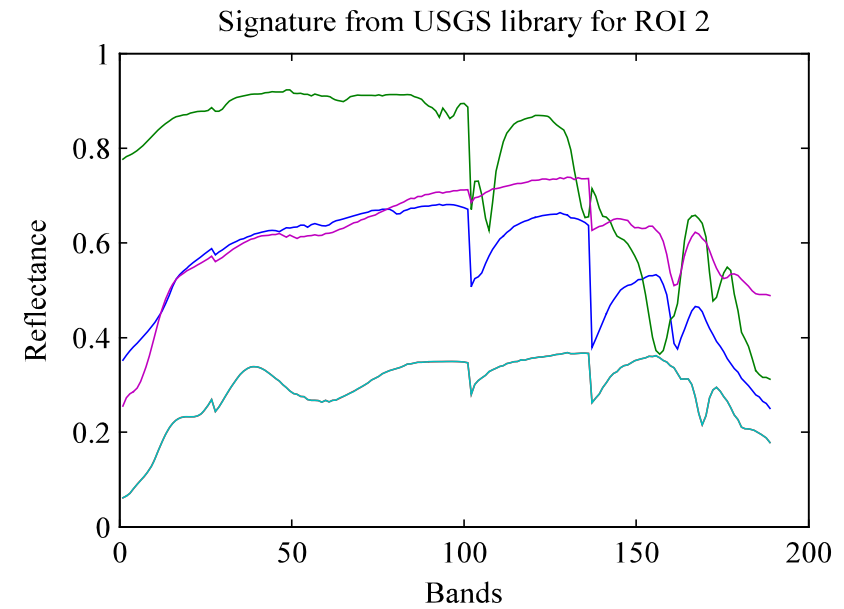

Figure 11. Matched signatures from USGS spectral library for ROI 2.

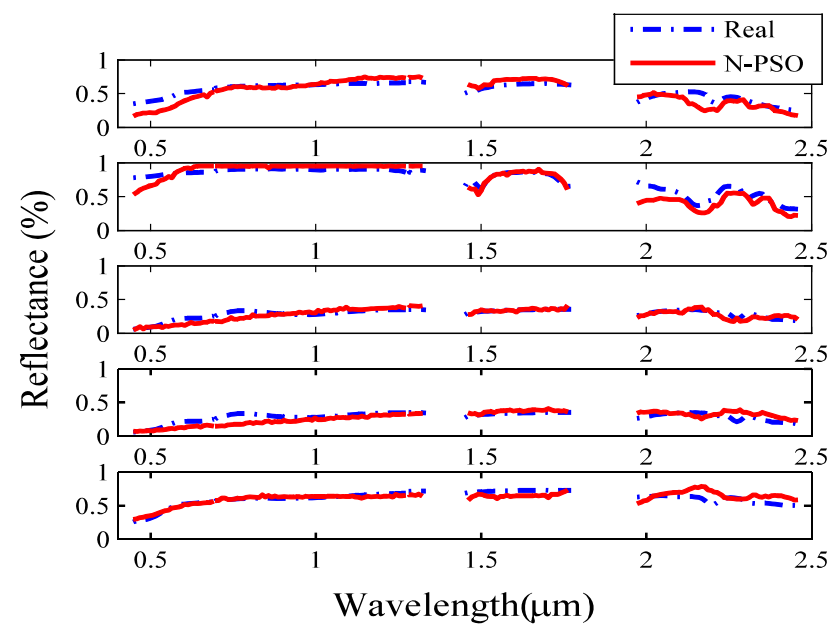

Figure 12. Graph showing similarity between the endmember signatures of real (extracted from USGS) and that extracted by N-PSO for ROI 2.

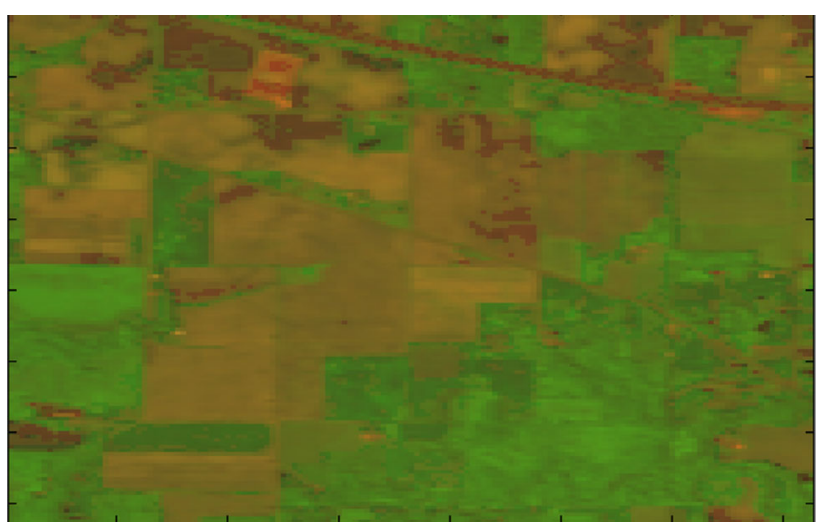

Figure 13. False colour composition of the Indian Pines data set with bands [13 54 97].

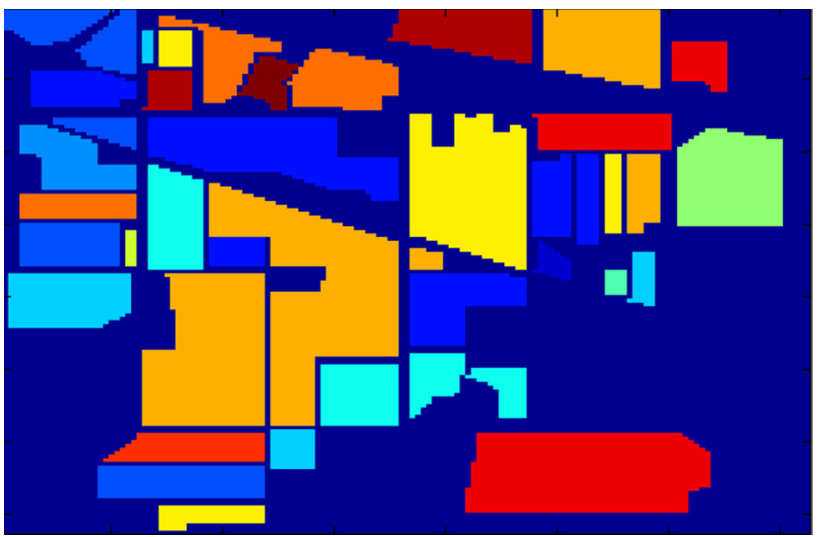

Figure 14. False colour composition of the ground truth of Indian Pines data set.

Table 6. Performance of N-PSO with other EEAs using the Indian Pines data set.

\begin{tabular}{lccc}
\hline$K=17$ & RE & SAM & Time \\
\hline VCA & 0.0904 & 0.1875 & $\mathbf{0 . 3 7 1 6}$ \\
DMaxD & 1.9976 & 0.3292 & 5995 \\
rNMF & 0.4745 & 0.7068 & 576 \\
N-PSO & $\mathbf{0 . 0 3 2 7}$ & $\mathbf{0 . 1 7 7 1}$ & 7025 \\
\hline
\end{tabular}

Best solutions are in bold.

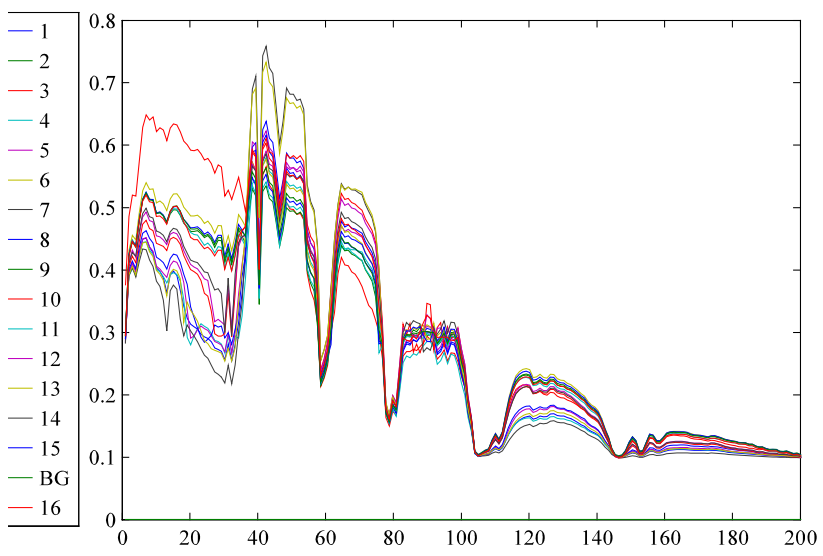

Figure 15. Endmembers extracted by averaging the data from the ground truth.

extracted from the original data for the experimentation. The ground truth of Cuprite data set is not available; therefore, the unmixing accuracies of the proposed N-PSO and another state-of-art algorithm are evaluated using RE. The value of $K$ is initialized to 5. As given in table 5, the N-PSO algorithm outperforms the other algorithms except for its time complexity. The estimated spectral signature of the endmembers extracted through the N-PSO algorithm for ROI 1 is given in figure 7. Their matched spectral 


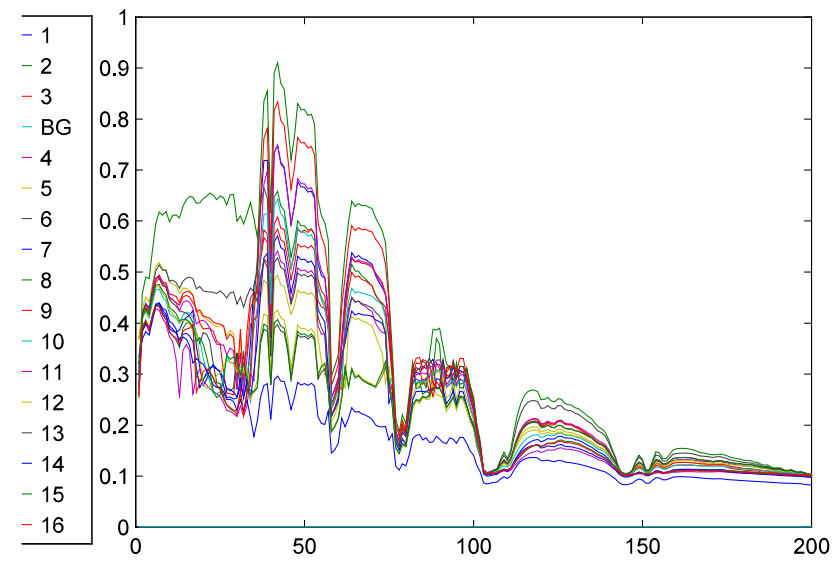

Figure 16. Endmembers extracted by VCA.

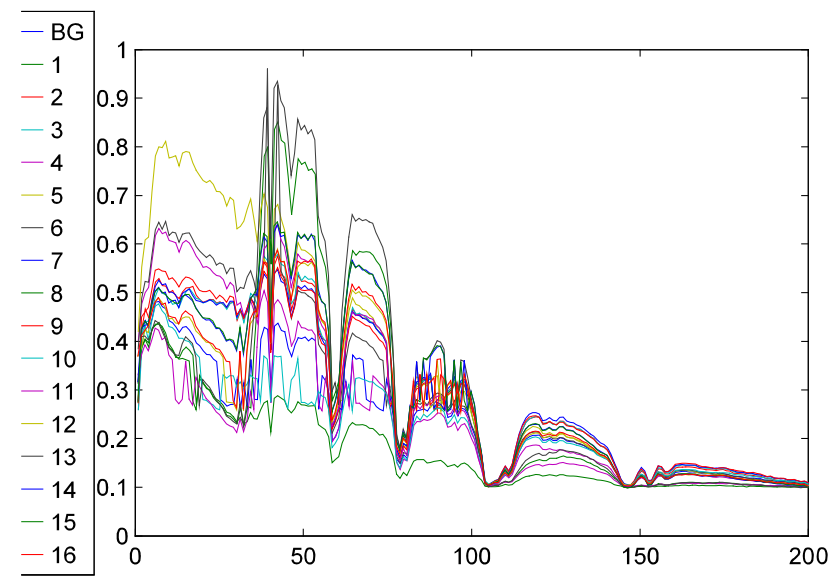

Figure 17. Endmembers extracted by DMaxD.

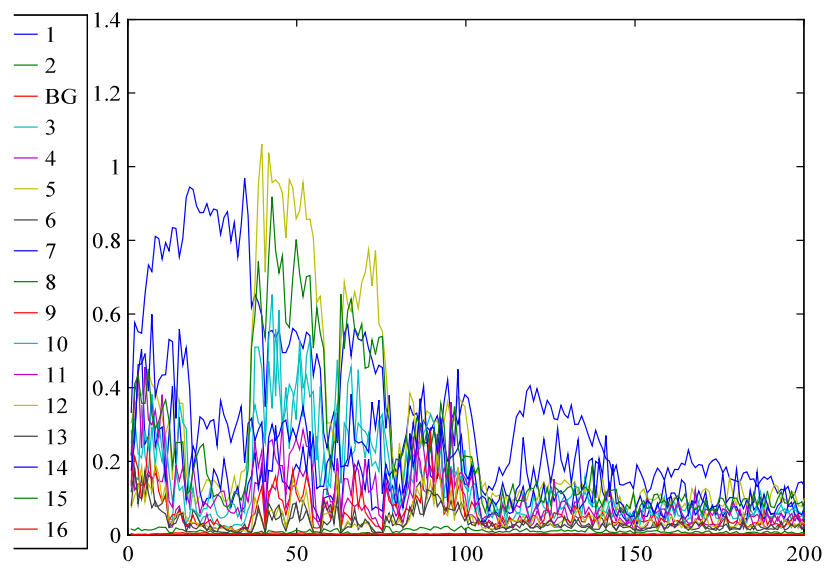

Figure 18. Endmembers extracted by rNMF.

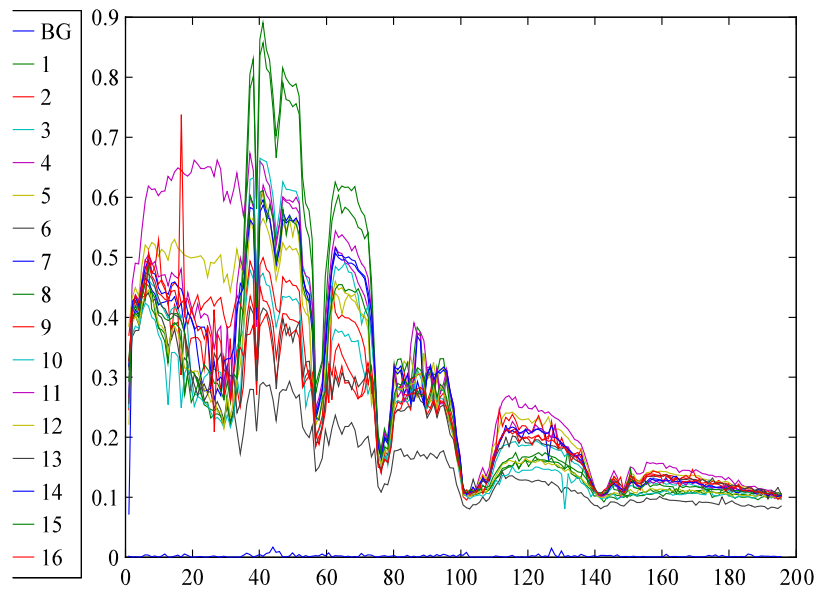

Figure 19. Endmembers extracted by N-PSO.

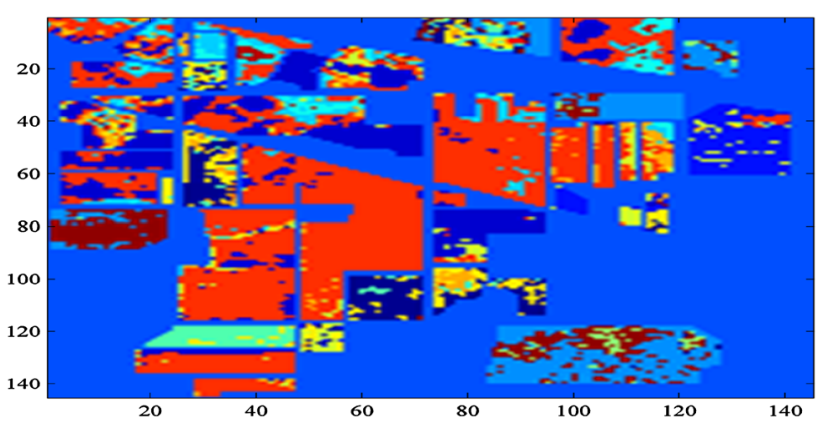

Figure 20. Classification of the Indian Pines data set using endmembers extracted by VCA.

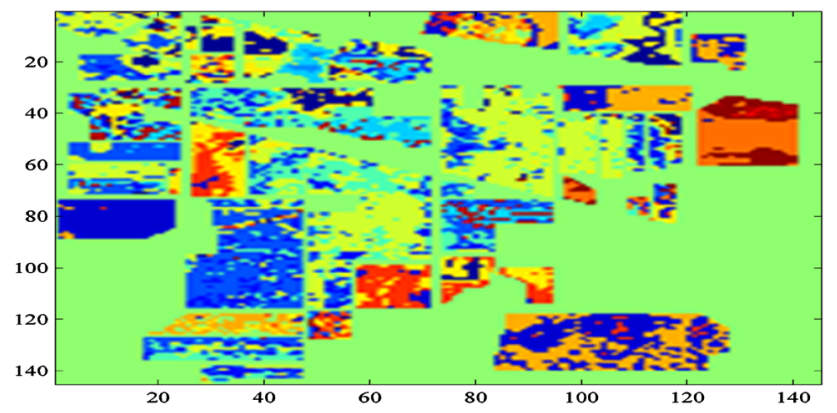

Figure 21. Classification of the Indian Pines data set using endmembers extracted by DMaxD.

signatures, namely those of Calcite WS272, Montmorillonite SCa-2.a Kaolin/Smect H89-FR-2 50K, Nontronite SWa-1.a and Nontronite NG-1.b $<2 \mu \mathrm{m}$ from USGS spectral library, are shown in figure 8 . The similarity between the endmembers extracted by N-PSO and matched spectral signature from USGS spectral library is shown in figure 9. Similarly, the spectral signature of endmembers by N-PSO, their matched spectral signature from USGS 


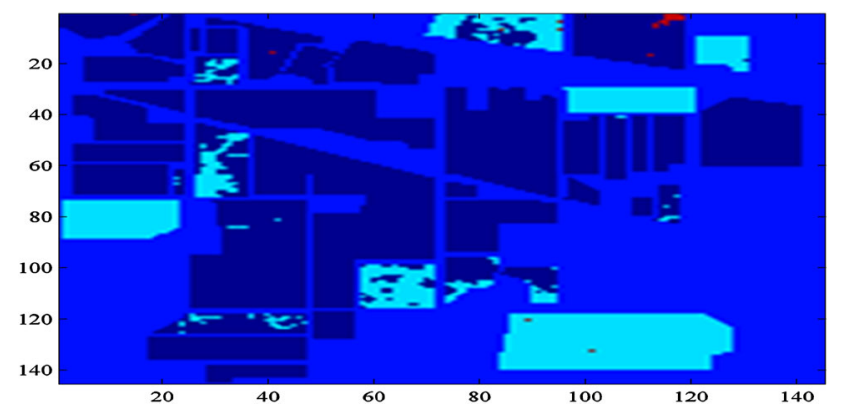

Figure 22. Classification of the Indian Pines data set using endmembers extracted by rNMF.

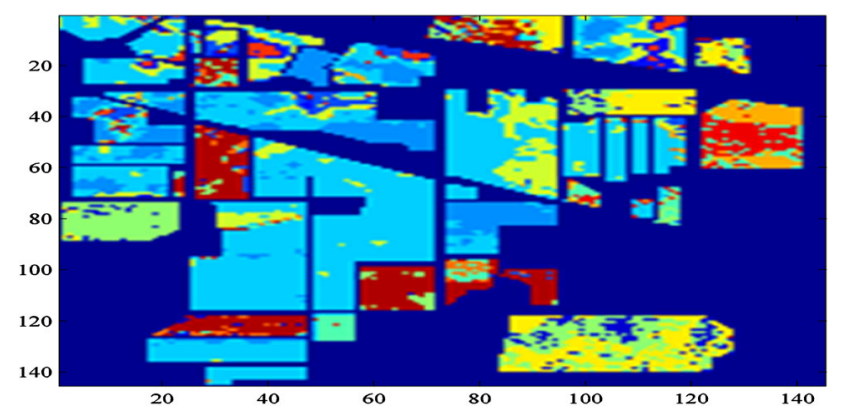

Figure 23. Classification of the Indian Pines data set using endmembers extracted by N-PSO.

spectral library (i.e., Montmorillonite SCa-2.b, Halloysite NMNH106236, Nontronite NG-1.b $<2 \mu \mathrm{m}$ fr, Nontronite NG-1.b $<2 \mu \mathrm{m}$ fr,and Montmorillonite SWy-1) and similarity graph are shown in figures 10, 11 and 12, respectively.

To evaluate the unmixing accuracy of the proposed N-PSO algorithm, the most widely used benchmark data set of Indian Pines (in figure 13) is used. The statistical significance of the results has been compared to those of the true endmembers extracted by averaging the set of the pixels of each class from the ground truth shown in figure 14. Only the pixels that are correctly classified as per the ground truth were considered for evaluation. The background information is eliminated from the original image for experimentation. The performance of N-PSO and another three EEAs is given in table 6. Looking at the final $\mathrm{RE}$ and SAM values given in table 6, we can say that a significant improvement regarding unmixing accuracy has been achieved by N-PSO. The endmembers extracted using the ground truth are shown in figure 15 . The resultant endmembers extracted using VCA, DMaxD, rNMF and N-PSO are shown in figures 16, 17, 18 and 19 respectively. The classification map using the abundances evaluated by the respective abundance estimation algorithm (i.e., FCLS in the case of VCA and DMaxD, SO in the case of N-PSO) for VCA, DMaxD, rNMF and N-PSO is shown in figures $20,21,22$ and 23 , respectively. The following steps are used to create the classification map of the respective data set using VCA, DMaxD, rNMF and N-PSO algorithms:

- calculate the abundance vector of all the pixels of a given image;

Table 7. Performance of FC-TLBO with FCLS and MCMC in terms of OA, AA and kappa for the Indian Pines data set.

\begin{tabular}{|c|c|c|c|c|c|c|c|c|c|c|}
\hline \multirow[b]{2}{*}{ Class } & \multirow[b]{2}{*}{ Class name } & \multirow[b]{2}{*}{ True samples } & \multicolumn{4}{|c|}{ Correctly estimated samples } & \multicolumn{4}{|c|}{ Class-wise accuracy (\%) } \\
\hline & & & VCA & DMaxD & rNMF & N-PSO & VCA & DMaxD & $\mathrm{rNMF}$ & N-PSO \\
\hline Class 1 & Alfalfa & 46 & 24 & 12 & 0 & 27 & 52.173 & 26.086 & 0 & 58.695 \\
\hline Class 2 & Corn-notill & 1428 & 1256 & 490 & 30 & 1250 & 87.955 & 34.313 & 2.100 & 87.535 \\
\hline Class 3 & Corn-mintill & 830 & 391 & 121 & 0 & 560 & 47.108 & 14.578 & 0 & 67.469 \\
\hline Class 4 & Corn & 237 & 64 & 17 & 0 & 123 & 27.004 & 7.1729 & 0 & 51.898 \\
\hline Class 5 & Grass-pasture & 483 & 309 & 156 & 0 & 371 & 63.975 & 32.298 & 0 & 76.811 \\
\hline Class 6 & Grass-trees & 730 & 124 & 230 & 0 & 430 & 16.986 & 31.506 & 0 & 58.904 \\
\hline Class 7 & Grass-pas-mowed & 28 & 12 & 8 & 0 & 17 & 42.857 & 28.571 & 0 & 60.714 \\
\hline Class 8 & Hay-windrowed & 478 & 129 & 203 & 0 & 224 & 26.987 & 42.468 & 0 & 46.861 \\
\hline Class 9 & Oats & 20 & 6 & 7 & 0 & 14 & 30 & 35 & 0 & 70 \\
\hline Class 10 & Soybean-notill & 972 & 570 & 458 & 0 & 630 & 58.641 & 47.119 & 0 & 64.814 \\
\hline Class 11 & Soybean-mintill & 2455 & 1568 & 734 & 1498 & 1045 & 63.869 & 29.898 & 61.018 & 42.566 \\
\hline Class 12 & Soybean-clean & 593 & 206 & 233 & 0 & 358 & 34.738 & 39.291 & 0 & 60.370 \\
\hline Class 13 & Wheat & 205 & 32 & 13 & 0 & 109 & 15.609 & 6.3414 & 0 & 53.170 \\
\hline Class 14 & Woods & 1265 & 790 & 609 & 1150 & 786 & 62.450 & 48.142 & 90.909 & 62.134 \\
\hline Class 15 & Build-Gra-Tr-Driv & 386 & 201 & 140 & 0 & 281 & 52.072 & 36.269 & 0 & 72.797 \\
\hline \multirow[t]{4}{*}{ Class 16} & Stone-Steel-Tow & 93 & 11 & 32 & 0 & 45 & 11.827 & 34.408 & 0 & 48.387 \\
\hline & & & & & & $\mathrm{OA}$ & 43.391 & 30.841 & 9.626 & 61.445 \\
\hline & & & & & & AA & 55.546 & 33.788 & 26.129 & 61.176 \\
\hline & & & & & & KAPPA & 0.34 & 0.21 & 0.09 & 0.47 \\
\hline
\end{tabular}


- for a pixel, find the position of the greatest value from the respective abundance vector of size $(K, 1)$;

- the position of the greatest value defines the pixel class;

- assign the colour of the respective endmember for the given pixel;

- repeat these three steps for each pixel of a given image;

- display the classification map.

The RE and SAM of N-PSO are 0.0327 and 0.1711, respectively, which are the best amongst the other approaches. However, its execution time is the largest among the other state-of-art algorithms.

To evaluate the performance of classification, overall accuracy (OA), average accuracy (AA) and kappa coefficient are used as an evaluation parameter [39]. In detail, to evaluate the performance of classification accuracy the statistical significance of the results has been computed. All the classification results that have been obtained using VCA, DMaxD, rNMF and N-PSO algorithm depend on the values of their respective abundance fractions. The dominant values among the abundances of the particular pixel are used to classify the pixel to the respective class.

The classification results obtained using the Indian Pines data set is reported in table 7 . The classifier used for an experiment depends on the spectral value of a pixel. Therefore, only the pixels that were correctly classified according to the ground truth were considered for the evaluation of classification. Comparing the final OA, AA and kappa coefficient values after application of the proposed N-PSO and other algorithms, a significant improvement regarding classification accuracy has been achieved in most of the cases for the Indian Pines data set. As shown in table 7 , the OA values using VCA, DMaxD, rNMF and N-PSO are $43.391 \%, 30.841 \%, 9.626 \%$ and $61.445 \%$, respectively, whereas the kappa coefficients are $0.34,0.21$, 0.09 and 0.43 , respectively.

\subsection{Inferences from the results of the synthetic and real data}

The results of the experiments on synthetic and real data lead to the following inferences:

- As regards the other state-of-art algorithms, the time of execution of VCA is the best. However, VCA is designed only for LMM, and its unmixing accuracy is a matter of major concern.

- The DMaxD algorithm based on geometrical distance shows better accuracy than the VCA. However, its accuracy is not better than those of rNMF and N-PSO algorithms in most of the cases. In addition, DMaxD uses the PPNM model for distance calculation, where the nonlinearity parameter is considered as a known parameter. Thus, it is not included in a category of the fully unsupervised approach of endmember extraction.
- In the known environment, the unmixing accuracy of the rNMF algorithm is better than those of other approaches. However, 1. The results using the rNMF algorithm do not follow the constraints on the values of the endmembers.

2. Although the RE using rNMF algorithm is the smallest in most of the cases, the SAM calculated using rNMF is at its worst value.

- The fully unsupervised approach based on artificial intelligence for the endmember extraction using NLMM is proposed for the first time in literature. The results show that N-PSO is faster than traditional $\mathrm{PSO}$, it is independent of the prior initialization of control parameters and satisfies the constraints on the value of endmember set. Although the time complexity of proposed N-PSO algorithm is more than those of the other state-of-art algorithms, the unmixing accuracy is better than those of the existing approaches.

\section{Conclusion and perspective work}

The paper demonstrates an efficient implementation of the N-PSO algorithm for endmember extraction using NLMM in known and unknown environments. This algorithm is a representative method of a class of algorithms for endmember extraction using swarm intelligence. The experiments demonstrate that the N-PSO algorithm outperforms the other algorithms by achieving better accuracy in term of endmember extraction, classification and RE. The experimental analyses also reveal that the proposed algorithm is more robust to variation in SNR than other state-of-art algorithms.

Our prospective work is intended to reduce the computational complexity for the images of larger sizes containing more number of endmembers using high-performance computing platforms. In future research, we will focus on the implementation of N-PSO using parallel programming and graphics processing units (GPU).

\section{References}

[1] Goetz A, Vane G, Solomon J and Rock B 1985 Imaging spectrometry for earth remote sensing. Science 228: $1147-1153$

[2] Green R O, et al 1998 Imaging spectroscopy and the airborne visible-infrared imaging spectrometer (AVIRIS). Remote Sens. Environ. 65(3): 227-248

[3] Hapke B W 1981 Bidirectional reflectance spectroscopy: 1. Theory. J. Geophys. Res. 86(B4): 3039

[4] Heylen R, Parente M and Gader P 2014 A review of nonlinear hyperspectral unmixing methods. IEEE J. Sel. Topics Appl. Earth Observ. Remote Sens. 7(6): 1844-1865 
[5] Dobigeon N, et al 2013 Nonlinear unmixing of hyperspectral images. IEEE Signal Process. Mag. 31(1): 82-94.

[6] Chang C I and Plaza A 2006 A fast iterative algorithm for implementation of pixel purity index. IEEE Geosci. Remote Sens. Lett. 3(1): 63-67

[7] Winter M E 1999 N-FINDR: an algorithm for fast autonomous spectral end-member determination in hyperspectral data. In: Proceedings of the SPIE Conference on Imaging Spectrometry, Pasadena, CA, pp. 266-275

[8] Nascimento J and Bioucas-Dias J 2015 Vertex component analysis: a fast algorithm to unmix hyperspectral data. IEEE Trans. Geosci. Remote Sens. 43(4): 898-910

[9] Li J and Bioucas-Dias J 2008 Minimum volume simplex analysis: a fast algorithm to unmix hyperspectral data. In: Proceedings of the IEEE International Geoscience and Remote Sensing Symposium, IGARSS2008, Boston,

[10] Ambikapathi A, Chan T H, Ma W K and Chi C Y 2011 Chance constrained robust minimum volume enclosing simplex algorithm for hyperspectral unmixing. IEEE Trans. Geosci. Remote Sens. 49(11): 4194-4209

[11] Chan T H, Ma W K, Ambikapathi A and Chi C Y 2011 A simplex volume maximization framework for hyperspectral endmember extraction. IEEE Trans. Geosci. Remote Sens. 49(11): 4177-4193

[12] Bioucas-Dias J 2009 A variable splitting augmented Lagrangian approach to linear spectral unmixing. In: Proceedings of the IEEE Workshop on Hyperspectral Image and Signal Processing: Evolution in Remote Sensing, pp. 1-4

[13] Zhang B, Gao J, Gao L and Sun X 2013 Improvements in ant colony optimization algorithm for endmember extraction from hyperspectral images. IEEE J. Sel. Topics Appl. Earth Observ. Remote Sens. 6(2): 522-530

[14] Zhang B, Sun X, Gao L and Yang L 2011 Endmember extraction of hyperspectral remote sensing images based on the discrete particle swarm optimization algorithm. IEEE Trans. Geosci. Remote Sens. 49(11): 4173-4176

[15] Ghamisi P and Benediktsson J A 2013 Feature selection based on hybridization of genetic algorithm and particle swarm optimization. IEEE Trans. Geosci. Remote Sens. 12(2): 309-313

[16] Zhong Y, Zhao L, and Zhang L 2014 An adaptive differential evolution endmember extraction algorithm for hyperspectral remote sensing imagery. IEEE Geosci. Remote Sens. Lett. 11(6): 1061-1065

[17] Heylen R, Scheunders P, Rangarajan A and Gader P 2015 Nonlinear unmixing by using different metrics in a linear unmixing chain. IEEE J. Sel. Topics Appl. Earth Observ. Remote Sens. 8(6): 2655-2664

[18] Ammanouil R, Ferrari A, Richard C and Mathieu S 2017 Nonlinear unmixing of hyperspectral data with vector-valued kernel functions. IEEE Trans. Image Process. 26(1): 340-354

[19] Févotte C and Dobigeon N 2015 Nonlinear hyperspectral unmixing with robust nonnegative matrix factorization. IEEE Trans. Image Process. 24(12): 4810-4819

[20] Mogale D G, Kumar S K, Mrquez F P G and Tiwari M K 2017 Bulk wheat transportation and storage problem of public distribution system. Comput. Ind. Eng. 104(C): 80-97

[21] Maiyar L M and Thakakr J J, 2017 A combined tactical and operational deterministic food grain transportation model: particle swarm based optimization approach. Comput. Ind. Eng. 110: 30-42

[22] Maiyar L M, Thakakr J J, Awasthi A and Tiwari M K 2015 Development of an effective cost minimization model for food grain shipments. IFAC-PapersOnLine 48(3): 881-886

[23] Mogale D G, Dolgui A, Kandhway R, Kumar S K and Tiwari M K 2017 A multi-period inventory transportation model for tactical planning of food grain supply chain. Comput. Ind. Eng. 110: 379-394

[24] De A, Mamanduru V K R, Gunasekaran A, Subramanian N and Tiwari M K 2016 Composite particle algorithm for sustainable integrated dynamic ship routing and scheduling optimization. Comput. Ind. Eng. 96: 201-215

[25] De A, Kumar S K, Gunasekaran A and Tiwari M K 2017 Sustainable maritime inventory routing problem with time window constraints. Eng. Appl. Artif. Intell. 61: 77-95

[26] De A, Awasthi A and Tiwari M K 2017 Robust formulation for optimizing sustainable ship routing and scheduling problem. IFAC-PapersOnLine 48(3): 368-373

[27] Chen M C, Hsiao Y H, Reddy R H and Tiwari M K 2016 The self-learning particle swarm optimization approach for routing pickup and delivery of multiple products with material handling in multiple cross-docks. Transport. Res. E: Logist. Transport. Rev. 91: 208-226

[28] Bioucas-Dias J M and Nascimento J M P 2008 Hyperspectral subspace identification. IEEE Trans. Geosci. Remote Sens. 46(8): 2435-2445

[29] Altmann Y, Halimi A, Dobigeon N and Tourneret J Y 2012 Supervised nonlinear spectral unmixing using a postnonlinear mixing model for hyperspectral imagery. IEEE Trans. Image Process. 21(6): 3017-3025

[30] Kennedy J and Eberhart R C 1995 Particle swarm optimization. In: Proceedings of the IEEE International Conference on Neural Networks, Perth, Australia, pp. 1942-1948

[31] Heinz D C, et al 2001 Fully constrained least squares linear spectral mixing analysis method for material quantification in hyperspectral imagery. IEEE Trans. Geosci. Remote Sens. 39(3): 529-545

[32] http://www.lx.it.pt/ bioucas/code.htm. Visiting date: 10 May 2016

[33] http://speclab.cr.usgs.gov/spectral-lib.html. Visiting date: 12 May 2016

[34] http://aviris.jpl.nasa.gov/data/free_data.html. Visiting date: 1 June 2016

[35] http://www.ehu.eus/ccwintco/index.php?title=Hyperspectral_ Remote. Visiting date: 1 June 2016

[36] http://www.lx.it.pt/ bioucas/code.htm. Visiting date: 2 Dec 2016

[37] https://sites.google.com/site/robheylenresearch/code. Visiting date: 2 Jan 2017

[38] https://www.irit.fr/ Cedric.Fevotte/publications.html. Visiting date: 3 Jan 2017

[39] Bakos K, Marpu P R and Gamba P 2011 Decision fusion of multiple classifiers for hyperspectral data classification. In: Prasad S, Bruce L M and Chanussot J (Eds.) Optical remote sensing: advances in signal processing and exploitation techniques, 1st ed. Augmented vision and reality. New York: Springer-Verlag 